\title{
Prognostic value of coronary computed tomography angiography in diabetic patients without chest pain syndrome
}

\author{
Inge J. van den Hoogen, BSc, ${ }^{\mathrm{a}}$ Michiel A. de Graaf, MSc, ${ }^{\mathrm{a}, \mathrm{b}}$ Cornelis J. Roos, \\ $M D,{ }^{a, b}$ Aukelien C. Leen, MD, ${ }^{a}$ Aan V. Kharagjitsingh, MD, PhD, ${ }^{\mathrm{c}}$ \\ Ron Wolterbeek, MD, ${ }^{\mathrm{d}}$ Lucia J. Kroft, $M D$, PhD, ${ }^{\mathrm{e}}$ J. Wouter Jukema, $M D, \mathrm{PhD},{ }^{\mathrm{a}, \mathrm{b}}$ \\ Jeroen J. Bax, MD, PhD, ${ }^{a}$ and Arthur J. Scholte, $M D, P^{a}$ \\ a Department of Cardiology, Leiden University Medical Center, Leiden, The Netherlands \\ ${ }^{\mathrm{b}}$ The Interuniversity Cardiology Institute of the Netherlands, Utrecht, The Netherlands \\ c Department of Internal Medicine, Westeinde Hospital, The Hague, The Netherlands \\ d Department of Medical Statistics and Bio-informatics, Leiden University Medical Center, \\ Leiden, The Netherlands \\ e Department of Radiology, Leiden University Medical Center, Leiden, The Netherlands
}

Received Feb 23, 2015; accepted May 11, 2015

doi: 10.1007/s12350-015-0213-5

Aims. Diabetic patients with coronary artery disease (CAD) are often free of chest pain syndrome. A useful modality for non-invasive assessment of CAD is coronary computed tomography angiography (CTA). However, the prognostic value of CAD on coronary CTA in diabetic patients without chest pain syndrome is relatively unknown. Therefore, the aim was to investigate the long-term prognostic value of coronary CTA in a large population diabetic patients without chest pain syndrome.

Methods. Between 2005 and 2013, 525 diabetic patients without chest pain syndrome were prospectively included to undergo coronary artery calcium (CAC)-scoring followed by coronary CTA. During follow-up, the composite endpoint of all-cause mortality, non-fatal myocardial infarction (MI), and late revascularization ( $>90$ days) was registered.

Results. In total, CAC-scoring was performed in 410 patients and coronary CTA in 444 patients (431 interpretable). After median follow-up of 5.0 (IQR 2.7-6.5) years, the composite endpoint occurred in $65(14 \%)$ patients. Coronary CTA demonstrated a high prevalence of CAD $(85 \%)$, mostly non-obstructive CAD $(51 \%)$. Furthermore, patients with a normal CTA had an excellent prognosis (event-rate 3\%). An incremental increase in event-rate was observed with increasing CAC-risk category or coronary stenosis severity. Finally, obstructive (50-70\%) or severe CAD $(>70 \%)$ was independently predictive of events (HR 11.10 [2.52;48.79] $(P=.001)$, HR 15.16 $[3.01 ; 76.36](P=.001))$. Obstructive $(50-70 \%)$ or severe CAD $(>70 \%)$ provided increased value over baseline risk factors.

Conclusion. Coronary CTA provided prognostic value in diabetic patients without chest pain syndrome. Most importantly, the prognosis of patients with a normal CTA was excellent. (J Nucl Cardiol 2016;23:24-36.)

Key Words: Computed tomography (CT) • diagnostic and prognostic application • diabetes • atherosclerosis

Inge J. van den Hoogen and Michiel A. de Graaf share first authorship Reprint requests: Michiel A. de Graaf, Department of Cardiology, Leiden University Medical Center, Albinusdreef 2, Postal zone 2300 RC, 2333 ZA Leiden, The Netherlands; M.A.de_Graaf@lumc.nl
$1071-3581 / \$ 34.00$

Copyright (C) 2015. The Author(s). This article is published with open access at Springerlink.com 


\begin{tabular}{|ll|}
\hline Abbreviations & \\
CABG & Coronary artery bypass grafting \\
CAC & Coronary artery calcium \\
CAD & Coronary artery disease \\
CTA & Computed tomography angiography \\
DM & Diabetes mellitus \\
ESC & European Society of Cardiology \\
MI & Myocardial infarction \\
PCI & Percutaneous coronary intervention \\
\hline
\end{tabular}

\section{See related editorial, pp. 37-41}

\section{INTRODUCTION}

Diabetes mellitus (DM) is a major and rapidly growing global health problem. In 2013, DM was responsible for $8.4 \%$ of all-cause mortality in patients between 20 and 79 years old and $10.8 \%$ of total health expenditure worldwide. ${ }^{1}$ Cardiovascular complications are the leading cause of mortality in diabetic patients. ${ }^{2}$ Accordingly, the European Society of Cardiology (ESC) classifies patients with $\mathrm{DM}$ as high risk for coronary artery disease (CAD). ${ }^{3}$ However, not all patients with DM have CAD and also diabetic patients with $\mathrm{CAD}$ are often free of chest pain syndrome. ${ }^{4}$ Coronary computed tomography angiography (CTA) is a useful modality for non-invasive assessment of CAD. Indeed, in diabetic patients without chest pain syndrome, a high prevalence of CAD is present on coronary CTA ${ }^{5-7}$ Potentially CTA could be used to risk stratify DM patients. However, the prognostic value of $\mathrm{CAD}$ on coronary CTA in these patients is relatively unknown. As a consequence, the value of coronary CTA for risk stratification of these patients is unestablished. ${ }^{8,9}$ Therefore, the aim of this study is to investigate the long-term prognostic value of coronary CTA in a large population of diabetic patients without chest pain syndrome.

\section{METHODS}

\section{Patients}

The study population consisted of 525 diabetic patients without chest pain syndrome, referred from an outpatient diabetic clinic for assessment of cardiovascular risk between May 2005 and August 2013. The cardiovascular assessment includes coronary artery calcium (CAC) score and CTA to evaluate the presence and severity of CAD. ${ }^{5,10}$ After enrolment in the prospective clinical registry, patients underwent a noncontrast CT for CAC-scoring followed by a contrast coronary CTA. Inclusion criteria consisted of confirmed diagnosis of
DM type 1 or 2 (fasting plasma glucose level $\geq 126 \mathrm{mg} / \mathrm{dL}$, use of oral glucose lowering medication or insulin) and the absence of chest pain syndrome. ${ }^{11}$ Exclusion criteria were known or suspected (CAD), previous coronary revascularization, cardiac arrhythmias, pregnancy, and contraindications for the use of iodinated contrast media.

Clinical data were prospectively entered into the departmental Cardiology Information System (EPD-Vision@), Leiden University Medical Center, the Netherlands) and retrospectively analyzed. The Institutional Review Board of the Leiden University Medical Center approved this evaluation of clinically collected data, and waived the need for written informed consent.

\section{Coronary CTA Acquisition}

Patients were scanned using a 64-slice or 320-row multidetector scanner (64-slice: Aquillon 64, Toshiba Medical Systems, Otawara, Japan; 320-row: Aquillon ONE, Toshiba Medical System, Otawara, Japan). Scan-protocol was followed as previously described. ${ }^{12,13}$ Post-processing of scans was performed with application of dedicated software (Vitrea FX 1.0, Vital Images, Minnetonka, MN, USA). Uninterpretable scans were excluded from the analysis.

\section{CAC-Scoring}

CAC-scoring was performed according to the algorithm of Agatston. CAC-score was stratified into four risk categories: $0,1-99,100-399, \geq 400 .^{12}$

\section{Coronary CTA}

All coronary CTAs were analyzed by consensus of experienced observers according to the modified 17 segments American Heart Association (AHA) classification.

First, each segment was assessed for interpretability. Segments were defined as uninterpretable in case of severe motion artefacts or low-contrast resolution. Additionally, segments with a diameter $\leq 1.5 \mathrm{~mm}$ were excluded. ${ }^{5}$ Second, interpretable segments were evaluated for stenosis. Stenosis was stratified into four categories: normal if no plaques were present on CTA, nonobstructive if the plaque covered $<50 \%$, obstructive if the plaque covered $50-70 \%$, severe if the plaque covered $>70 \%$ of the coronary artery lumen. If plaque was present, plaque composition was determined (calcified, mixed, and non-calcified). One type of plaque compositions was assigned per segment.

\section{Follow-Up}

Follow-up data were retrospectively gathered by review of electronic medical records; blinded from CTA results, between December 2013 and February 2014, both the medical records of the department of cardiology and of the referring outpatient diabetic clinic have been analyzed. Three endpoints were registered: all-cause mortality, non-fatal myocardial infarction (MI), late revascularization. Non-fatal MI was defined based on criteria of typical chest pain, elevated cardiac enzyme levels, and typical changes on the ECG. ${ }^{14}$ Late revascularization was 
defined as percutaneous coronary intervention (PCI) or coronary artery bypass grafting (CABG) after 90 days of scan acquisition. ${ }^{15}$ All revascularization procedures within 90 days were considered coronary CTA-driven. For the analysis, a composite endpoint was constructed of all three endpoints.

\section{Statistical Analysis}

All continuous data (normally distributed, non-normally distributed) are presented as mean \pm SD for reasons of uniformity. Categorical data are presented as absolute numbers and percentages.

First, baseline characteristics were compared between patients with and without obstructive CAD ( $\geq 50 \%)$, similarly between patients with and without events. Second, results of both CAC-scoring and coronary CTA were compared between patients with and without events. Third, survival analyses were performed by the Kaplan-Meier method. Cumulative eventrates for CAC-score and coronary stenosis were obtained by this method, using the composite endpoint. Note that these survival analyses were crude, because no corrections for baseline characteristics were performed. Fourth, the independent prognostic value of baseline characteristics, CAC-scoring, and coronary CTA was assessed. For this purpose, univariate and multivariate Cox-regression analyses were performed. To avoid over fitting of the model, a selection of univariate significant variables was entered into the multivariate model.

All statistical tests were two-sided. Comparisons between groups were performed with the Independent-Samples $T$ test or Mann-Whitney $U$ test for continuous data and the $\chi^{2}$ test for categorical data. Comparisons of Kaplan-Meier curves were performed with the Log-Rank test. To compare the model fit of the multivariate Cox-regression models for CTA and CAC-score the -2 Log Likelihood was used. However, it should be noted that for non-nested models (i.e., Model 2 vs. Model 4), this only provides a crude comparison for which no $P$ values could be calculated. All statistical analyses were performed with SPSS software (Version 22.0, SPSS IBM Corp., Armonk, New York). A $P$ value $<.05$ was considered statistically significant.

\section{RESULTS}

\section{Patients}

The study population consisted of 525 patients. As depicted in Figure 1, $76(14 \%)$ patients were excluded from this analysis because of logistical reasons (i.e., patients who did not attend appointment). The results of 449 patients were available for the present analysis: 405 patients underwent both CAC-scoring and coronary CTA; 5 patients underwent only CAC-scoring; 39 patients underwent only coronary CTA. In total, CACscoring was performed in 410 patients and coronary CTA in 444 patients. Mean age was $54 \pm 11$ years; 265 (59\%) patients were male, and median DM duration was 12 (IQR 6-22) years. Baseline characteristics of the population are depicted in Table 1.

\section{Events}

The composite endpoint of all-cause mortality, nonfatal MI, and late revascularization occurred in $65(14 \%)$ patients. All-cause mortality occurred in 13 (3\%)

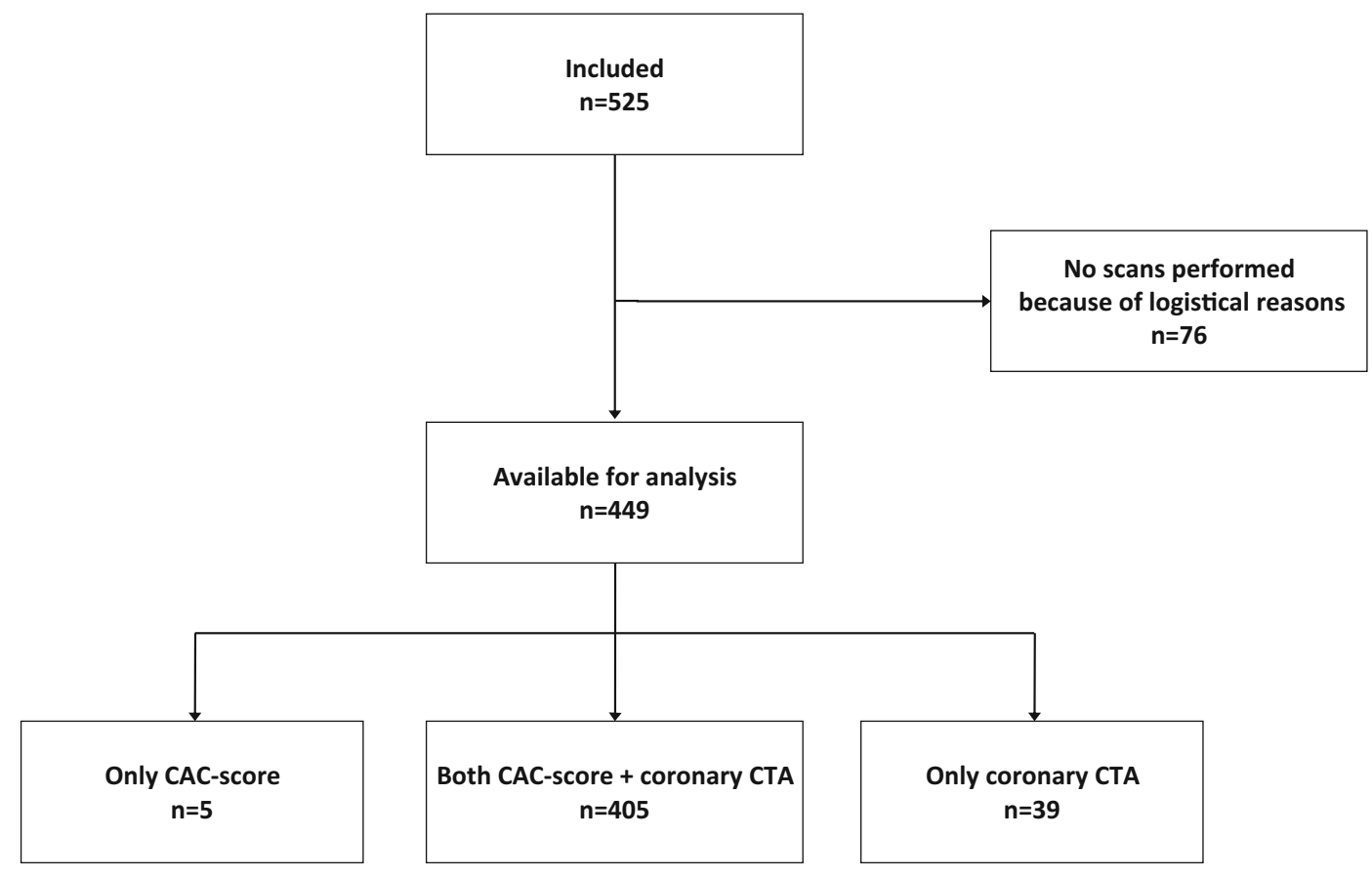

Figure 1. Flowchart of the study population. 


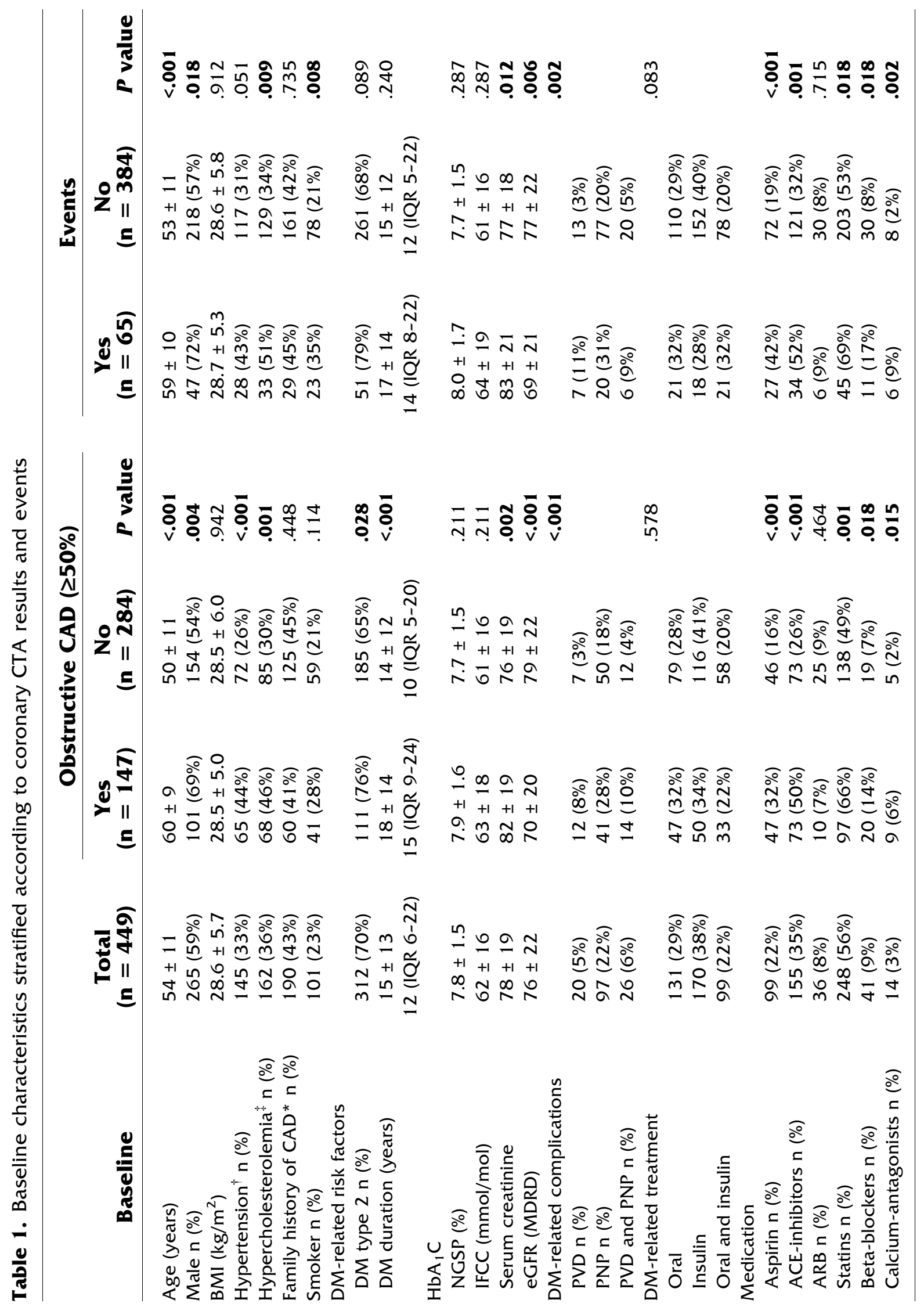


patients and late revascularization in $52(12 \%)$ patients (PCI: 30 patients, CABG: 22 patients). Of the 52 patients who underwent revascularization, 27 (52\%) patients were referred to invasive coronary angiography because of new-onset angina, $20(38 \%)$ patients had documented ischemia on a single positron emission tomography (SPECT) myocardial perfusion imaging (MPI) performed after CTA, and 5 (10\%) patients presented with new ischemia on SPECT MPI during follow-up. Non-fatal MI did not occur. Early revascularization, which was excluded from the composite endpoint, occurred in $16(4 \%)$ patients (PCI: 13 patients, CABG: 3 patients). Median follow-up was 5.0 (IQR 2.7-6.5) years. No patients were lost to follow-up.

Patients with events demonstrated a higher mean age $[59 \pm 10$ vs. $53 \pm 11(P<.001)]$ and number of males [47 (72\%) vs. $218(57 \%)(P=.018)]$ compared to patients without events. Furthermore, hypercholesterolemia $\left[\begin{array}{lllll}33 & (51 \%) & \text { vs. } 129 & (34 \%) & (P=.009)\end{array}\right]$, smoker [23 (35\%) vs. $78(21 \%)(P=.008)]$, and overall DM-related complications $(P=.002)$ were more frequently observed in this group (Table 1).

\section{CAC-Scoring}

CAC-scoring was performed in 410 patients. Median CAC-score was 29 (IQR 0-294). The distribution within the CAC-risk categories was as follows: CAC-score $=0$ in $144(35 \%)$ patients, CAC-score $=1-99$ in $106(26 \%)$ patients, CAC-score $=100-399$ in $67(16 \%)$ patients, CAC-score $\geq 400$ in $93(23 \%)$ patients. In total, CACscore $=0$ and CAC-score $\geq 1$ were observed in respectively $35 \%$ and $65 \%$ of patients (Table 2).

The results of CAC-scoring, stratified according to events, are depicted in Table 2. Patients with events demonstrated a higher median CAC-score compared to patients without events [543 (IQR 141-1310) vs. 13 (IQR 0-177) $(P<.001)]$. Moreover, patients with events were more often classified in a higher CAC-risk category $(P<.001)$.

\section{Coronary CTA}

Coronary CTA was performed in 444 patients, of which 13 were uninterpretable. The remaining results of 431 patients were used for the present analysis. A high prevalence of $\mathrm{CAD}(85 \%)$ was demonstrated on coronary CTA: non-obstructive CAD $(<50 \%)$ in $219(51 \%)$ patients, obstructive CAD (50-70\%) in 117 (27\%) patients, severe CAD (>70\%) in $30(7 \%)$ patients. A normal CTA was observed in $65(15 \%)$ patients (Table 2).

The baseline characteristics, stratified according to coronary CTA results, are depicted in Table 1. Patients with obstructive CAD $(\geq 50 \%)$ demonstrated a higher 
Table 2. Results of CAC-scoring and coronary CTA stratified according to events

\begin{tabular}{|c|c|c|c|c|c|}
\hline \multirow[b]{2}{*}{ CAC-scoring } & \multirow[b]{2}{*}{$\begin{array}{c}\text { Total } \\
(\mathrm{n}=\mathbf{4 1 0})\end{array}$} & \multicolumn{4}{|c|}{ Events } \\
\hline & & $\begin{array}{c}\text { Yes } \\
(n=59)\end{array}$ & \multicolumn{2}{|c|}{$\begin{array}{c}\text { No } \\
(n=351)\end{array}$} & $P$ value \\
\hline CAC-score & $\begin{array}{c}355 \pm 800 \\
29(\text { IQR 0-294) }\end{array}$ & $\begin{array}{c}1043 \pm 1449 \\
543(\text { IQR } 141-1310)\end{array}$ & \multicolumn{2}{|c|}{$\begin{array}{c}239 \pm 554 \\
13(\mathrm{IQR} 0-177)\end{array}$} & $<.001$ \\
\hline \multicolumn{5}{|l|}{ CAC-risk category } & $<.001$ \\
\hline CAC-score $=0$ & $144(35 \%)$ & $5(9 \%)$ & \multicolumn{2}{|c|}{$139(40 \%)$} & \\
\hline CAC-score $=1-99$ & $106(26 \%)$ & $7(12 \%)$ & \multicolumn{2}{|c|}{$99(28 \%)$} & \\
\hline CAC-score $=100-399$ & $67(16 \%)$ & $12(20 \%)$ & \multicolumn{2}{|c|}{$55(16 \%)$} & \\
\hline \multirow[t]{2}{*}{ CAC-score $\geq 400$} & $93(23 \%)$ & 35 (59\%) & \multicolumn{2}{|c|}{$58(17 \%)$} & \\
\hline & & & & Events & \\
\hline \multicolumn{2}{|c|}{ Coronary CTA } & $\begin{array}{c}\text { Total } \\
(n=431)\end{array}$ & $\begin{array}{c}\text { Yes } \\
(n=64)\end{array}$ & $\begin{array}{c}\text { No } \\
(n=367)\end{array}$ & $P$ value \\
\hline \multicolumn{5}{|l|}{ Coronary stenosis } & $<.001$ \\
\hline \multicolumn{3}{|c|}{ No. of patients with normal CTA n (\%) } & $2(3 \%)$ & $63(17 \%)$ & \\
\hline \multicolumn{2}{|c|}{ No. of patients with non-obstructive CAD $(<50 \%)$ n (\%) } & $219(51 \%)$ & $11(17 \%)$ & $208(57 \%)$ & \\
\hline \multicolumn{2}{|c|}{ No. of patients with obstructive CAD (50-70\%) n (\%) } & $117(27 \%)$ & $39(61 \%)$ & $78(21 \%)$ & \\
\hline \multicolumn{2}{|c|}{ No. of patients with severe CAD (>70\%) n (\%) } & $30(7 \%)$ & $12(19 \%)$ & $18(5 \%)$ & \\
\hline \multicolumn{6}{|c|}{ Coronary plaques (stenosis) } \\
\hline \multicolumn{2}{|c|}{ No. of plaques } & $8.0 \pm 5.6$ & $9.7 \pm 4.4$ & $7.7 \pm 5.7$ & .002 \\
\hline \multicolumn{2}{|c|}{ No. of non-obstructive lesions } & $7.0 \pm 5.3$ & $6.9 \pm 4.1$ & $7.1 \pm 5.4$ & .724 \\
\hline \multicolumn{2}{|c|}{ No. of obstructive lesions } & $1.0 \pm 1.9$ & $2.8 \pm 2.8$ & $0.6 \pm 1.4$ & $<.001$ \\
\hline \multicolumn{2}{|l|}{ No. of severe lesions } & $0.1 \pm 0.5$ & $0.3 \pm 0.8$ & $0.1 \pm 0.4$ & .009 \\
\hline \multicolumn{6}{|c|}{ Coronary plaques (composition) } \\
\hline \multicolumn{2}{|c|}{ No. of calcified lesions } & $1.1 \pm 2.2$ & $2.6 \pm 3.5$ & $0.8 \pm 1.8$ & $<.001$ \\
\hline \multicolumn{2}{|l|}{ No. of mixed lesions } & $1.5 \pm 2.4$ & $3.0 \pm 3.0$ & $1.3 \pm 2.1$ & $<.001$ \\
\hline \multicolumn{2}{|c|}{ No. of non-calcified lesions } & $0.9 \pm 1.5$ & $1.3 \pm 2.2$ & $0.8 \pm 1.4$ & .067 \\
\hline
\end{tabular}

Bold values are statistically significant $(P<0.05)$

$C A C$, Coronary artery calcium; $C A D$, Coronary artery disease; $C T A$, Computed tomography coronary angiography

mean age $[60 \pm 9$ vs. $50 \pm 11(P<.001)]$, number of males $[101(69 \%)$ vs. $154(54 \%)(P=.004)]$, and median diabetes duration [15 (IQR 9-24) vs. 10 (IQR 5-20) $(P<.001))$ compared to patients with no or nonobstructive CAD $(<50 \%)$. Furthermore, hypertension [65 (44\%) vs. $72(26 \%)(P<.001)]$, hypercholesterolemia $[68(46 \%)$ vs. $85(30 \%)(P=.001)]$, and overall DM-related complications $(P<.001)$ were more frequently observed in this group.

The results of coronary CTA, stratified according to events, are depicted in Table 2. Patients with events presented with more severe coronary stenosis compared to patients without events $(P<.001)$. Moreover, a higher mean number of plaques $[9.7 \pm 4.4$ vs. $7.7 \pm 5.7$ $(P=.002)]$, mean number of obstructive lesions $[2.8 \pm 2.8$ vs. $0.6 \pm 1.4(P<.001)]$, and mean number of severe lesions $[0.3 \pm 0.8$ vs. $0.1 \pm 0.4(P=.009)]$ were observed in this group. In addition, a higher mean number of calcified lesions $[2.6 \pm 3.5$ vs. $0.8 \pm 1.8$
$(P<.001)]$ and mixed lesions $[3.0 \pm 3.0$ vs. $1.3 \pm 2.1$ $(P<.001)]$ were present in patients with events.

\section{Kaplan-Meier Analysis}

The results of the Kaplan-Meier survival analyses, stratified according to CAC-score, are depicted in Figure $2 \mathrm{~A}$ and $\mathrm{B}$. Crude event-rate was lower in patients with CAC-score $=0$ compared to patients with CACscore $\geq 1[5 / 144(3 \%)$ vs. 54/266 (20\%) $(P<.001)]$ (A). Additionally, an incremental increase in event-rate was observed with increasing CAC-risk category: 5/144 (3\%) for CAC-score $=0,7 / 106(7 \%)$ for CAC-score $=1-99$, $12 / 67$ (18\%) for CAC-score $=100-399,35 / 93(38 \%)$ for CAC-score $\geq 400 \quad(P<.001)$. Thus, event-rate was highest in patients with CAC-score $\geq 400$ (B).

The results of the Kaplan-Meier survival analyses, stratified according to coronary stenosis, are depicted in Figure $2 \mathrm{C}$ and D. Crude event-rate was lower in patients 
A

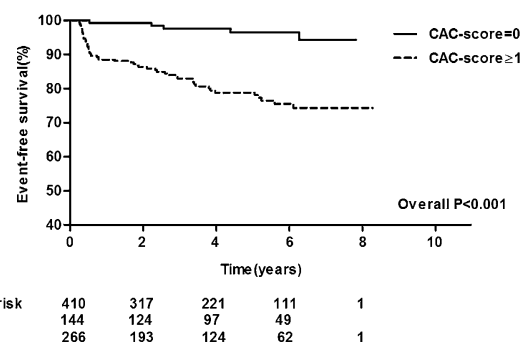

B

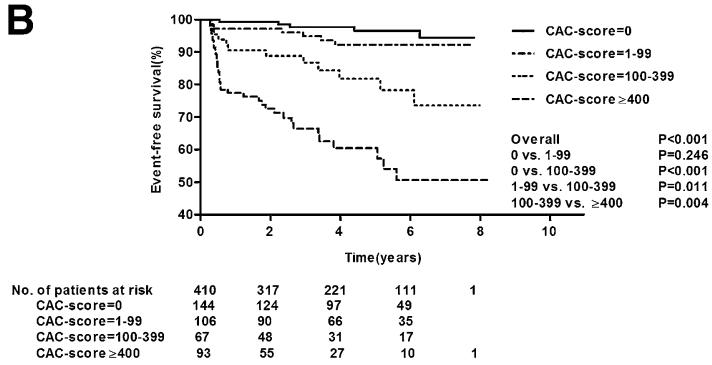

D
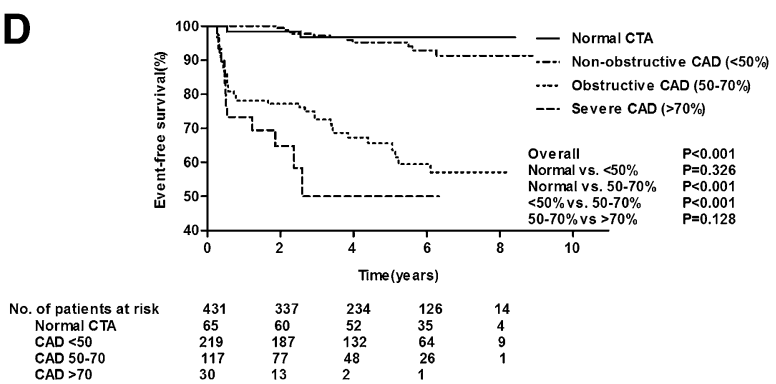

Figure 2. Kaplan-Meier curves for the composite endpoint (all-cause mortality, non-fatal MI, late revascularization) according to CAC-score and coronary stenosis. (A) Event-free survival difference between patients with CAC-score $=0$ and CAC-score $\geq 1$. (B) event-free survival difference between patients with CAC-score $=0$, CAC-score $=1-99$, CAC-score $=100-399$ and CACscore $\geq 400$. (C) event-free survival difference between patients with no or non-obstructive CAD $(<50 \%)$ and obstructive CAD $(\geq 50 \%)$. (D) event-free survival difference between patients with normal CTA, non-obstructive CAD $(<50 \%)$, obstructive CAD (50$70 \%)$ and severe $\mathrm{CAD}(>70 \%)$.

with no or non-obstructive CAD $(<50 \%)$ compared to patients with obstructive CAD $(\geq 50 \%)[13 / 284(5 \%)$ vs. $51 / 147(35 \%)(P<.001)](C)$. An excellent prognosis was observed in patients with a normal CTA [event-rate $2 / 65(3 \%)$ ]. Of note, the 2 patients with a normal CTA presented with complications not related to diabetes and died of a (presumably) non-cardiac course. Additionally, an incremental increase in event-rate was observed with increasing coronary stenosis severity: 11/219 (5\%) for non-obstructive $\mathrm{CAD}(<50 \%), \quad 39 / 117 \quad(33 \%)$ for obstructive CAD (50-70\%), 12/30 (40\%) for severe CAD $(>70 \%)(P<.001)$. Event-rate was highest in patients with severe CAD (>70\%) (D).

\section{Cox-Regression Analysis}

The results of univariate Cox-regression analyses for the prediction of events are depicted in Tables 3 and 4 . CAC-score $\geq 100$, obstructive (50-70\%) or severe CAD $(>70 \%)$, total number of plaques, number of plaques stratified to stenosis (obstructive, severe) and number of plaques stratified to plaque composition (calcified, mixed, non-calcified) were all significant univariate predictors of the composite endpoint (Table 4).

The results of the multivariate Cox-regression analyses for the prediction of events are depicted in
Table 5. To avoid over fitting of the model (limited number of events, $n=65$ ), only a selection of the univariate significant variables was entered into the multivariate model (i.e., age, male gender, smoker, CAC-risk category, coronary stenosis grade). The variable smoker was selected over hypertension and hypercholesterolemia, assuming it correlated less with age and male gender. Moreover, replacing smoking with either hypertension or hypercholesterolemia did not have a strong influence on the results. In the multivariate analyses, corrected for the selected baseline variables (Model 2), CAC-score $\geq 100$ was independently predictive of events. Moreover, CAC-score $\geq 400$ provided incremental prognostic value over CAC-score $=100$ 399 [HR $12.52(95 \%$ CI $4.29 ; 36.54)(P<.001)$ vs. HR 5.13 (95\% CI $1.68 ; 15.60)(P=.004)]$. Accordingly, obstructive $(50-70 \%)$ or severe CAD $(>70 \%)$ remained an independent predictor of events. The presence of severe CAD $(>70 \%)$ provided incremental prognostic value over obstructive CAD (50-70\%) [HR 15.16 (95\% CI 3.01 ; 76.36) $(P=.001)$ vs. HR 11.10 (95\% CI 2.52; 48.79) $(P=.001)]$. Adding the CTA results to Model 2 (including baseline variables and CAC-score) resulted in a significant change in -2 Log Likelihood (17.60, $P=.001$ ) (Model 3). Moreover, adding the CTA results to the baseline model resulted in a larger increase in the 
Table 3. Univariate Cox-regression analyses of baseline characteristics for the prediction of events

\begin{tabular}{lcc}
\hline \multicolumn{1}{c}{ Baseline } & $\begin{array}{c}\text { Univariate } \\
\text { HR [95\%CI] }\end{array}$ & $\boldsymbol{P}$ value \\
\hline Age & $1.06[1.04 ; 1.09]$ & $<.001$ \\
Male & $1.97[1.14 ; 3.38]$ & $\mathbf{. 0 1 5}$ \\
BMI & $1.00[0.96 ; 1.04]$ & .959 \\
Hypertension & $1.79[1.10 ; 2.93]$ & $\mathbf{. 0 2 0}$ \\
Hypercholesterolemia & $2.15[1.32 ; 3.51]$ & $\mathbf{. 0 0 2}$ \\
Family history of CAD & $0.95[0.58 ; 1.56]$ & .846 \\
Smoker & $1.98[1.19 ; 3.30]$ & $\mathbf{. 0 0 8}$ \\
DM-related risk factors & & \\
DM type 2 & $1.58[0.87 ; 2.85]$ & .132 \\
DM duration & $1.00[1.00 ; 1.00]$ & .160 \\
HbA ${ }_{1}$ C & $1.08[0.92 ; 1.27]$ & .326 \\
DM-related complications & & \\
PVD & $3.01[1.37: 6.60]$ & $\mathbf{. 0 0 6}$ \\
PNP & $1.66[0.98 ; 2.82]$ & .058 \\
PVD and PNP & $1.77[0.76 ; 4.10]$ & .184 \\
DM-related treatment & & \\
Oral & $1.13[0.67 ; 1.90]$ & .646 \\
Insulin & $0.60[0.35 ; 1.04]$ & .069 \\
Oral and insulin & $1.79[1.06 ; 3.01]$ & $\mathbf{. 0 2 8}$ \\
Medication & & \\
Aspirin & $2.78[1.69 ; 4.55]$ & $<.001$ \\
ACE-inhibitors & $2.39[1.47 ; 3.89]$ & $<.001$ \\
ARB & $1.03[0.44 ; 2.39]$ & .946 \\
Statins & $1.97[1.16 ; 3.34]$ & $\mathbf{. 0 1 2}$ \\
Beta-blockers & $2.29[1.20 ; 4.39]$ & $\mathbf{. 0 1 2}$ \\
Calcium-antagonists & $3.94[1.70 ; 9.14]$ & $\mathbf{. 0 0 1}$ \\
Serum markers & & \\
Total cholesterol & $1.23[0.99 ; 1.54]$ & .067 \\
LDL & $1.13[0.89 ; 1.43]$ & .317 \\
HDL & $0.92[0.55 ; 1.54]$ & .756 \\
Cholesterol/HDL ratio & $1.06[0.93 ; 1.22]$ & .364 \\
Triglycerides & $1.09[0.91 ; 1.31]$ & .364 \\
\hline Bold & & \\
\hline
\end{tabular}

Bold values are statistically significant $(P<0.05)$

Abbreviations and definitions as in Table 1

-2 Log Likelihood compared to the adding of the CACscore (43.78 vs. 36.30) (Model 4).

\section{DISCUSSION}

The present study assessed the long-term prognostic value of coronary CTA in a large prospective registry of diabetic patients without chest pain syndrome. Coronary CTA demonstrated high prevalence of CAD (85\%), mostly non-obstructive. Most importantly, patients with a normal CTA had an excellent prognosis. Furthermore, an incremental increase in event-rate was observed with increasing coronary stenosis severity. Finally, obstructive $(50-70 \%)$ or severe CAD $(>70 \%)$ was independently predictive of events, with increased value over baseline risk factors (i.e., age, male gender, smoker). Moreover, the CAC-score demonstrated a similar independent predictive value for the occurrence of events. However, the model including CTA performed better than the model with CAC-score. Besides, CTA provided some additional value over the CAC-score. Nevertheless, it should be noted that this was a crude analysis and that the present study was not designed to assess the difference in performance between CAC-score and CTA.

\section{CAC-Score}

Previous studies widely established the prevalence of CAC in diabetic patients without chest pain syndrome. ${ }^{16-18}$ The present study assessed the prognostic value of CAC by demonstrating CAC-score $\geq 100$ as independent predictor of events in diabetic patients without chest pain syndrome. Prior to our study, Raggi et al investigated the prognostic value of CAC-scoring for all-cause mortality in asymptomatic individuals. ${ }^{19} \mathrm{In}$ this study, 10,377 asymptomatic individuals were prospectively included to undergo electron beam computed tomography (EBCT): 903 (9\%) individuals with DM, 9474 (91\%) individuals without DM. This study, with mean follow-up of 5 years, demonstrated CAC as independent predictor of all-cause mortality in both diabetic and non-diabetic asymptomatic individuals. Moreover, Anand et al investigated the prognostic value of EBCT for short-term events in 510 asymptomatic patients with DM type $2 .{ }^{20}$ This study, with median follow-up of 2.2 years, demonstrated CAC-score $\geq 100$ as independent predictor of cardiac death, MI, acute coronary syndrome (ACS), late coronary revascularization ( $>60$ days after EBCT), and non-haemorrhagic stroke over established cardiovascular risk factors. Additionally, the PREDICT (prospective evaluation of diabetic ischemic disease by computed tomography) study investigated the prognostic value of EBCT for cardiovascular events in 589 asymptomatic patients with DM type $2 .{ }^{21}$ Cardiovascular events, which were defined as death due to MI or other cardiovascular causes, nonfatal MI, unstable angina, other objective evidence of $\mathrm{CAD}$, and stroke, occurred in $66(11 \%)$ patients after median follow-up of 4 years. In the multivariate analyses, CAC-score $\geq 101$ was independently predictive of cardiovascular events. These findings were in line with the present study. Moreover, similar to the present study, incremental prognostic value was provided with increasing CAC-risk category. 
Table 4. Univariate Cox-regression analyses of CAC-scoring and coronary CTA for the prediction of events

\title{
Univariate
}

HR [95\%CI]

$P$ value

\author{
CAC-scoring \\ CAC-risk category \\ CAC-score $=0$ \\ CAC-score $=1-99$ \\ CAC-score $=100-399$ \\ CAC-score $\geq 400$ \\ Coronary CTA \\ Coronary stenosis \\ Normal CTA \\ Non-obstructive CAD (<50\%) \\ Obstructive CAD (50-70\%) \\ Severe CAD (>70\%) \\ Coronary plaques (stenosis) \\ No. of plaques \\ No. of non-obstructive lesions \\ No. of obstructive lesions \\ No. of severe lesions \\ Coronary plaques (composition) \\ No. of calcified lesions \\ No. of mixed lesions \\ No. of non-calcified lesions
}

$\begin{array}{lr}\text { Overall } & <.001 \\ \text { Ref. category } & .265 \\ 1.92[0.61 ; 6.05] & .001 \\ 6.11[2.15 ; 17.35] & <.001 \\ 15.79[6.16 ; 40.50] & \\ & <.001 \\ \text { Overall } & \\ \text { Ref. category } & .397 \\ 1.92[0.43 ; 8.67] & <.001 \\ 16.18[3.90 ; 67.21] & <.001 \\ 29.03[6.40 ; 131.73] & \\ & <.001 \\ 1.09[1.04 ; 1.14] & .375 \\ 1.02[0.98 ; 1.07] & <.001 \\ 1.40[1.31 ; 1.51] & <.001 \\ 2.42[1.77 ; 3.30] & \\ & <.001 \\ 1.22[1.14 ; 1.30] & <.001 \\ 1.24[1.16 ; 1.33] & \mathbf{0 0 0 7} \\ 1.17[1.04 ; 1.31] & \end{array}$

Bold values are statistically significant $(P<0.05)$

Abbreviations and definitions as in Table 2

\section{Coronary Stenosis on CTA}

Several large cohort studies assessed the prevalence of CAD in the specific setting of diabetic patients without chest pain syndrome. ${ }^{6,17,22,23}$ Similar to the present study, in these studies, the majority of asymptomatic diabetic patients presented with $\mathrm{CAD}$ on coronary CTA (64-93\%). Accordingly, non-obstructive CAD $(<50 \%)$ was most frequently observed (44-64\%), whereas obstructive CAD $(\geq 50 \%)$ was less prevalent (17-29\%).

Only a few studies assessed the prognostic value of coronary CTA in diabetic patients without chest pain syndrome. ${ }^{15,22,24}$ From the CONFIRM (coronary CT angiography evaluation for clinical outcomes: an international multicentre) registry of 27,125 patients, Min et al selected 400 asymptomatic diabetic patients who underwent coronary CTA. ${ }^{15}$ The prognostic value of CTA was investigated using the same composite endpoint as in the present study. Events occurred in $33(8 \%)$ patients after mean follow-up of $2.4 \pm 1.1$ years: allcause mortality in $13(3 \%)$ patients, non-fatal MI in 8 (2\%) patients, late revascularization in $12(3 \%)$ patients. In the multivariate analyses, corrected for selected variables (i.e., age, male gender, $\mathrm{CAC}$-score), maximal stenosis severity, number of vessels with obstructive $\mathrm{CAD}(\geq 50 \%)$, and segment stenosis score (a marker of overall atherosclerosis extent) were independently predictive of events. Indeed, obstructive (50-70\%) or severe $(>70 \%)$ CAD provided prognostic value in the present study. Also Faustino et al investigated the prognostic value of coronary CTA for cardiovascular events in 85 asymptomatic patients with DM type $2 .{ }^{24}$ Cardiovascular events occurred in $10(11.8 \%)$ patients after median follow-up of 48 (IQR 18-68) months: cardiovascular death in $2(2.4 \%)$ patients, unstable angina in $1(1.2 \%)$ patients, stroke $7(8.4 \%)$ in patients. In the multivariate analyses, corrected for univariate significant variables, the absence of obstructive CAD ( $\geq 50 \%)$ was independently protective of events. Indeed, no or nonobstructive CAD $(<50 \%)$ was not associated with increased risk for events in the present study. Most importantly, patients with a normal CTA had an excellent prognosis. Last, Park et al investigated the prognostic value of coronary CTA for cardiovascular events in 557 asymptomatic Korean patients with DM type 2. ${ }^{22}$ Cardiovascular events were defined as 


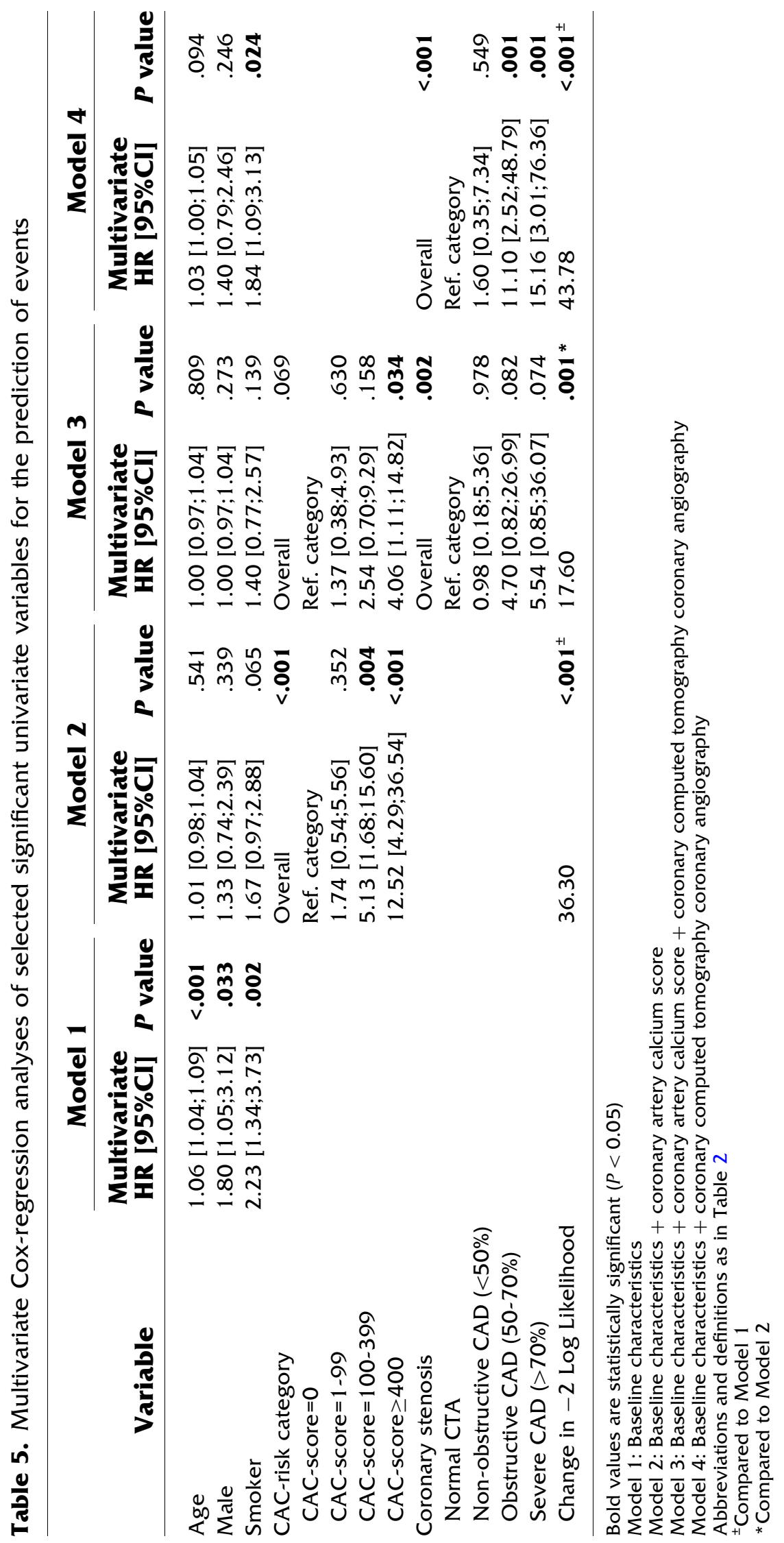


cardiovascular death, non-fatal MI, ACS requiring hospitalization, and late revascularization ( $>6$ months after coronary CTA). More cardiovascular events and lower 3 years event-free survival rates were observed in patients with obstructive CAD $(\geq 50 \%)$ compared to patients without obstructive CAD $(<50 \%)$. Accordingly, in the present study, a higher crude event-rate was observed in patients with obstructive CAD $(\geq 50 \%)$ compared to patients with no or non-obstructive CAD $(<50 \%)$.

\section{Coronary Plaque Composition on CTA}

Multiple studies assessed plaque composition on coronary CTA in diabetic patients without chest pain syndrome. Comparable to the present study, the majority of these studies described an increased prevalence of mixed lesions in asymptomatic diabetic patients. ${ }^{6,17,25}$

The prognostic value of coronary plaque composition on coronary CTA for cardiovascular events in the specific setting of diabetic patients without chest pain syndrome has not been previously established. The present study demonstrated all coronary plaque compositions (calcified, mixed, non-calcified) as univariate significant predictors of events. The prognostic value of calcified and mixed lesions was highest. These findings suggest an independent association between plaque composition and events.

However, the role of coronary plaque composition remains controversial. Gaemperli et al demonstrated the prognostic value of coronary plaque composition in 220 symptomatic patients. ${ }^{26}$ In contrast to the present study, mixed and non-calcified plaque provided the highest predictive value for events. On the other hand, in the CONFIRM registry, including both symptomatic and asymptomatic patients with and without DM, calcified and mixed plaque provided the strongest predictive value. ${ }^{27}$ Further research is needed to understand the underlying pathophysiological mechanism of the different coronary plaque compositions.

\section{SPECT Myocardial perfusion Imaging (MPI)}

The role of SPECT MPI for screening for silent ischemia in asymptomatic diabetic patients has been previously addressed. ${ }^{20,28}$ Anand et al included 510 asymptomatic diabetic patients of whom 180 patients underwent SPECT MPI. ${ }^{20}$ In those patients, the eventrate was significantly increased with increasing ischemic burden on SPECT MPI demonstrating the value for risk stratification. The most important study in this field was the DIAD (detection of Ischemia in Asymptomatic Diabetics) study. $^{28}$ In this randomized controlled trial, 1123 asymptomatic diabetic participants were randomized to SPECT MPI or no screening. After a mean follow-up of 4.8 years, the incidence of cardiac events was higher in patients with significant MPI abnormalities. However, there was no significant prognostic benefit of screening.

\section{Clinical Implications}

The present observations demonstrate the prognostic value of CTA in diabetic patients without chest pain syndrome. Recent ESC guidelines indicate patients with DM as high risk for CAD (or very high risk if $\geq 1$ cardiovascular risk factor was present) irrespective of chest pain symptoms. Indeed, in the present study, a great majority of patients presented with CAD on coronary CTA and the presence of obstructive $(50-70 \%)$ or severe CAD (>70\%) was associated with an impaired prognosis. Still, CAD was ruled-out in $15 \%$ of patients based on a normal CTA. Most importantly, the prognosis of these patients was excellent.

The value of screening for CAD in high-risk diabetic patients without chest pain syndrome was recently addressed by Muhlestein et al. ${ }^{29}$ In this trial, 900 asymptomatic patients were randomized to CAD screening using coronary CTA or optimal medical treatment (OMT). The trial demonstrated no survival benefit from screening with coronary CTA. Therefore, this study does not support screening in all diabetic patients. Similarly, the American Diabetes Association position statement on cardiovascular disease and risk management only recommend screening using advanced cardiac testing in patients with cardiac symptoms or ECG abnormalities. ${ }^{30}$ However, as also demonstrated in the present study, a large proportion of the patients had CAD on CTA and coronary CTA could identify patients with excellent prognosis. This supports the need to enrich the screening population to a high-risk population who will mostly benefit from screening using coronary CTA. Especially since coronary CTA may lead to radiation exposure and may result in unnecessary invasive testings such as coronary angiography and revascularization procedures. ${ }^{30}$ Potentially, coronary CTA can provide a pivotal role in tailored therapy in these diabetic patients. Patients with a normal CTA have an excellent prognosis and could be treated conservatively (OMT), whereas patients with an abnormal CTA may benefit from additional non-invasive or invasive evaluation.

\section{LIMITATIONS}

Several limitations of the present study need to be considered. First, the present study was a single-centre 
study. Second, the composite event-rate was relatively low. As a consequence, the study was underpowered to include all baseline risk factors into the multivariate model. Third, the endpoint mainly consisted of late revascularization; therefore, conclusions regarding hard endpoints are not justified based on this study. We cannot rule out that, despite the wide time interval, some referral bias has occurred in the patients who underwent late revascularization. Moreover, it is possible that events that have occurred in other medical centres were missed in the analysis. Fourth, coronary CTA only visualizes coronary atherosclerosis and provides no information on the hemodynamic significance of coronary stenosis.

\section{CONCLUSION}

Coronary CTA provided prognostic value in a large prospective registry of diabetic patients without chest pain syndrome. Most importantly, the prognosis of patients with a normal CTA was excellent. In addition, an incremental increase in event-rate was observed with increasing CAC-risk category and coronary stenosis severity. The highest event-rate was observed in patients with severe CAD $(>70 \%)$. Both CAC-score and coronary stenosis severity were independently predictive of events, after correction for baseline risk factors.

\section{NEW KNOWLEDGE GAINED}

Coronary CTA can be of clinical value for risk stratification of patients with diabetes mellitus without chest pain syndrome. Especially, event-free survival is excellent in patients without coronary artery disease. The prognostic value of coronary CTA seems to outweigh the prognostic value of the coronary calcium score.

\section{Disclosures}

The department of Cardiology of the Leiden University Medical Center received research grants from Biotronik, Medtronic, Boston Scientific Corporation, St Jude Medical, Lantheus Medical Imaging and GE Healthcare.

\section{Open Access}

This article is distributed under the terms of the Creative Commons Attribution 4.0 International License (http:// creativecommons.org/licenses/by/4.0/), which permits unrestricted use, distribution, and reproduction in any medium, provided you give appropriate credit to the original author(s) and the source, provide a link to the Creative Commons license, and indicate if changes were made.

\section{References}

1. International Diabetes Federation. Diabetes e-Atlas. Brussels: International Diabetes Federation; 2013.

2. Morrish NJ, Wang SL, Stevens LK, Fuller JH, Keen H. Mortality and causes of death in the WHO Multinational Study of Vascular Disease in Diabetes. Diabetologia 2001;44:S14-21.

3. Ryden L, Grant PJ, Anker SD, Berne C, Cosentino F, Danchin N, et al. ESC guidelines on diabetes, pre-diabetes, and cardiovascular diseases developed in collaboration with the E. Diab Vasc Dis Res 2014;11:133-73.

4. Zellweger MJ, Hachamovitch R, Kang X, Hayes SW, Friedman JD, Germano G, et al. Prognostic relevance of symptoms versus objective evidence of coronary artery disease in diabetic patients. Eur Heart J 2004;25:543-50.

5. Scholte AJ, Schuijf JD, Kharagjitsingh AV, Jukema JW, Pundziute $\mathrm{G}$, van der Wall EE, et al. Prevalence of coronary artery disease and plaque morphology assessed by multi-slice computed tomography coronary angiography and calcium scoring in asymptomatic patients with type 2 diabetes. Heart 2008;94:290-5.

6. Zeina AR, Odeh M, Rosenschein U, Zaid G, Barmeir E. Coronary artery disease among asymptomatic diabetic and nondiabetic patients undergoing coronary computed tomography angiography. Coron Artery Dis 2008;19:37-41.

7. Djaberi R, Beishuizen ED, Pereira AM, Rabelink TJ, Smit JW, Tamsma JT, et al. Non-invasive cardiac imaging techniques and vascular tools for the assessment of cardiovascular disease in type 2 diabetes mellitus. Diabetologia 2008;51(9):1581-93.

8. Perrone-Filardi P, Achenbach S, Mohlenkamp S, Reiner Z, Sambuceti G, Schuijf JD, et al. Cardiac computed tomography and myocardial perfusion scintigraphy for risk stratification in asymptomatic individuals without known cardiovascular disease: a position statement of the Working Group on Nuclear Cardiology and Cardiac CT of the European Society of Cardiology. Eur Heart J 2011;32:1986-93, 93a, 93b.

9. Taylor AJ, Cerqueira M, Hodgson JM, Mark D, Min J, O'Gara P, et al. ACCF/SCCT/ACR/AHA/ASE/ASNC/NASCI/SCAI/SCMR 2010 appropriate use criteria for cardiac computed tomography. A report of the American College of Cardiology Foundation Appropriate Use Criteria Task Force, the Society of Cardiovascular Computed Tomography, the American College of Radiology, the American Heart Association, the American Society of Echocardiography, the American Society of Nuclear Cardiology, the North American Society for Cardiovascular Imaging, the Society for Cardiovascular Angiography and Interventions, and the Society for Cardiovascular Magnetic Resonance. J Am Coll Cardiol 2010;56:1864-94.

10. Djaberi R, Schuijf JD, Boersma E, Kroft LJ, Pereira AM, Romijn JA, et al. Differences in atherosclerotic plaque burden and morphology between type 1 and 2 diabetes as assessed by multislice computed tomography. Diabetes Care 2009;32:1507-12.

11. American Diabetes Association. Diagnosis and classification of diabetes mellitus. Diabetes Care 2008;31:S55-60.

12. van Werkhoven JM, Schuijf JD, Gaemperli O, Jukema JW, Kroft LJ, Boersma E, et al. Incremental prognostic value of multi-slice computed tomography coronary angiography over coronary artery calcium scoring in patients with suspected coronary artery disease. Eur Heart J 2009;30:2622-9.

13. de Graaf FR, Schuijf JD, van Velzen JE, Kroft LJ, de Roos A, Reiber JH, et al. Diagnostic accuracy of 320-row multidetector computed tomography coronary angiography in the non-invasive evaluation of significant coronary artery disease. Eur Heart $\mathbf{J}$ 2010;31:1908-15 
14. Steg PG, James SK, Atar D, Badano LP, Blomstrom-Lundqvist C, Borger MA, et al. ESC Guidelines for the management of acute myocardial infarction in patients presenting with ST-segment elevation. Eur Heart J 2012;33:2569-619.

15. Min JK, Labounty TM, Gomez MJ, Achenbach S, Al-Mallah M, Budoff MJ, et al. Incremental prognostic value of coronary computed tomographic angiography over coronary artery calcium score for risk prediction of major adverse cardiac events in asymptomatic diabetic individuals. Atherosclerosis 2014;232:298-304.

16. Khaleeli E, Peters SR, Bobrowsky K, Oudiz RJ, Ko JY, Budoff MJ. Diabetes and the associated incidence of subclinical atherosclerosis and coronary artery disease: implications for management. Am Heart J 2001;141:637-44.

17. Rivera JJ, Nasir K, Choi EK, Yoon YE, Chun EJ, Choi SI, et al. Detection of occult coronary artery disease in asymptomatic individuals with diabetes mellitus using non-invasive cardiac angiography. Atherosclerosis 2009;203:442-8.

18. Choi EK, Chun EJ, Choi SI, Chang SA, Choi SH, Lim S, et al. Assessment of subclinical coronary atherosclerosis in asymptomatic patients with type 2 diabetes mellitus with single photon emission computed tomography and coronary computed tomography angiography. Am J Cardiol 2009;104:890-6.

19. Raggi P, Shaw LJ, Berman DS, Callister TQ. Prognostic value of coronary artery calcium screening in subjects with and without diabetes. J Am Coll Cardiol 2004;43:1663-9.

20. Anand DV, Lim E, Hopkins D, Corder R, Shaw LJ, Sharp P, et al. Risk stratification in uncomplicated type 2 diabetes: prospective evaluation of the combined use of coronary artery calcium imaging and selective myocardial perfusion scintigraphy. Eur Heart J 2006;27:713-21.

21. Elkeles RS, Godsland IF, Feher MD, Rubens MB, Roughton M, Nugara $F$, et al. Coronary calcium measurement improves prediction of cardiovascular events in asymptomatic patients with type 2 diabetes: the PREDICT study. Eur Heart J 2008;29:2244-51.

22. Park GM, Lee SW, Cho YR, Kim CJ, Cho JS, Park MW, et al. Coronary computed tomographic angiographic findings in asymptomatic patients with type 2 diabetes mellitus. Am J Cardiol 2014;113:765-71.

23. Silva JD, Mota P, Coelho A, Catarino R, Leitao-Marques A. Incidence of subclinical atherosclerosis in asymptomatic type-2 diabetic patients: the potential of multi-slice computed tomography coronary angiography. Coron Artery Dis 2011;22:26-31.

24. Faustino A, Providencia R, Mota P, Barra S, Silva J, Fernandes A, et al. Can cardiac computed tomography predict cardiovascular events in asymptomatic type- 2 diabetics?: results of a long term follow-up. BMC Cardiovasc Disord. 2014;14:2.

25. Pundziute G, Schuijf JD, Jukema JW, van Werkhoven JM, Nucifora G, Decramer I, et al. Type 2 diabetes is associated with more advanced coronary atherosclerosis on multislice computed tomography and virtual histology intravascular ultrasound. J Nucl Cardiol 2009;16:376-83.

26. Gaemperli O, Valenta I, Schepis T, Husmann L, Scheffel H, Desbiolles L, et al. Coronary 64-slice CT angiography predicts outcome in patients with known or suspected coronary artery disease. Eur Radiol 2008;18:1162-73.

27. Hadamitzky M, Achenbach S, Al-Mallah M, Berman D, Budoff M, Cademartiri F, et al. Optimized prognostic score for coronary computed tomographic angiography: results from the CONFIRM registry (COronary CT Angiography EvaluatioN For Clinical Outcomes: An InteRnational Multicenter Registry). J Am Coll Cardiol 2013;62:468-76.

28. Young LH, Wackers FJ, Chyun DA, Davey JA, Barrett EJ, Taillefer $\mathrm{R}$, et al. Cardiac outcomes after screening for asymptomatic coronary artery disease in patients with type 2 diabetes: the DIAD study: a randomized controlled trial. JAMA 2009;301:1547-55.

29. Muhlestein JB, Lappe DL, Lima JA, Rosen BD, May HT, Knight $\mathrm{S}$, et al. Effect of screening for coronary artery disease using CT angiography on mortality and cardiac events in high-risk patients with diabetes: the FACTOR-64 randomized clinical trial. JAMA 2014;312:2234-43.

30. American Diabetes Association. Cardiovascular disease and risk management. Diabetes Care 2015;38:S49-57. 